\title{
可见光-远红外图像的多模态能见度深度学习模型
}

\author{
沈克成 ${ }^{1)}$, 施佺 ${ }^{1,2}$ ，王晗 1,2$)^{*}$ \\ 1) (南通大学信息科学与技术学院 南通 226019) \\ 2) (南通大学交通与土木工程学院 南通 226019) \\ (hanwang@ntu.edu.cn)
}

\begin{abstract}
摘 要: 为了增强能见度深度学习模型在小样本条件下的准确率和鲁棒性, 提出一种基于可见光-远红外图像的多模 态能见度深度学习方法. 首先, 利用图像配准获取视野范围与分辨率均相同的可见光-远红外输人图像对; 然后, 构 造三分支并行结构的多模态特征融合网络; 分别在可见光图像、远红外图像及其累加特征图中提取不同性质的大气 特征, 各分支的特征信息通过网络结构实现模态互补与融合; 最后在网络末端输出图像场景所对应的能见度的等级. 采用双目摄像机收集不同天气情况下的室外真实可见光-远红外图像作为实验数据，在不同性能指标、多角度下的实 验结果表明, 与传统单模态能见度深度学习模型相比, 多模态能见度模型可显著提高小样本条件下能见度检测的准 确率和鲁棒性.
\end{abstract}

关键词: 能见度检测; 深度学习模型; 可见光-远红外图像; 多模态融合网络

中图法分类号: TP391.41 DOI: 10.3724/SP.J.1089.2021.18420

\section{Multimodal Visibility Deep Learning Model Based on Visible-Infrared Image Pair}

\author{
Shen Kecheng ${ }^{1)}$, Shi Quan ${ }^{1,2)}$, and Wang $\operatorname{Han}^{1,2)^{*}}$ \\ 1) (School of Information Science and Technology, Nantong University, Nantong 226019) \\ 2) (School of Transportation and Civil Engineering, Nantong University, Nantong 226019)
}

\begin{abstract}
In order to enhance the robustness of the visibility deep learning model under a small training dataset, this paper proposes a multi-modal visibility deep learning model based on visible-infrared image pairs. Apart from conventional visibility deep learning models, the visible-infrared image pairs are used as observation data. First, raw data set is preprocessed to generate visible-infrared image pairs with identical resolution and view range using image registration. Then we construct a new convolutional neural network structure including three CNN streams, which are connected in parallel. The feature maps of each stream are extracted and fused from low layer to deep layer by propagation. Finally, the visibility range level is classified by softmax layer based on the output feature descriptor of full connected layer. The experimental results demonstrate that, compared with conventional visibility deep learning models, both accuracy and robustness are strongly enhanced using the proposed method, especially for small training datasets.
\end{abstract}

Key words: visibility detection; deep learning model; visible-infrared image; multi-model fusion network

能见度是将目标从背景中识别出的最大距离 ${ }^{[1]}$. 其变化取决于大气透明度：透明度愈差, 能见距离
愈小. 雾䨪等天气可导致大气浑浊、透明度变小及 能见度陡降 ${ }^{[2]}$, 是诱发交通事故的重要原因 ${ }^{[3]}$. 能

收稿日期：2020-06-02; 修回日期：2021-03-22. 基金项目：国家自然科学基金(61872425); 南通市科技面上项目(MS12019051). 沈克成(1995-), 男, 硕士研究生, 主要研究方向为多模态图像处理、能见度检测; 施佺(1975一), 男, 博士, 教授, 博士生导师, 主要 研究方向为智能交通、大数据分析; 王晗(1979-), 男, 博士, 硕士生导师, CCF 会员, 论文通讯作者, 主要研究方向为图像处理、深 度学习。 
见度检测可有效地监测大气环境，对潜在的交通 事故提供可靠的预警依据. 基于图像的能见度检 测方法因设备价格低廉、安装方便等优势，相关研 究迅速发展 ${ }^{[4]}$.

目前, 基于图像能见度检测的研究丰富多样. 可分为统计学模型 ${ }^{[5-7]}$ 与深度学习模型 ${ }^{[8-9]}$. 统计学 方法通常利用物理模型或者概率模型来估计能见 度值. 如, Hautiére 等 ${ }^{[10]}$ 利用 Koschmieder 定律求解 能见度值. Babari 等 ${ }^{[11]}$ 提出基于亮度值对比度的非 线性描述函数能见度估计方法. $\mathrm{Li}$ 等 ${ }^{[12]}$ 通过定义 晴朗场景比率权重来修正大气消光系数, 进而通 过大气散射模型计算能见度.

随着卷积神经网络 (convolutional neural networks, CNN)的广泛应用, 能见度深度学习模型亦 成为研究热点. $\mathrm{Li}$ 等 ${ }^{[13]}$ 通过 AlexNet 提取大气特征, 再利用广义回归神经网络(general regression neural networks, GRNN)预测能见度范围. 模型经香港天 文台 2016-10-2017-08 的 1003 幅 RGB 图像集训 练准确率为 $62.0 \%$. Yang 等 ${ }^{[14]}$ 将前向卷积网络与循 环网络相结合, 通过递归循环神经网络(recurrent convolutional networks, CNN-RNN)模型融合全局 和局部特征，采用支持向量机(support vector ma- chine, SVM)对图像的能见度等级排序, 尝试利用 相对评价方法提高模型对场景变化的鲁棒性. 经 37420 幅 RGB 网络图像训练准确率为 $82.2 \%$. Palvanov 等 ${ }^{[15]}$ 将 RGB 图像进行预处理, 将其在频 率域和伪色彩空间上的滤波结果增强观测数据的 多样性. 设计并行链接的卷积网络结构, 对观测数 据进行能见度距离的分类. 模型经韩国气象局 300 万幅 RGB 图像训练准确率为 $91.3 \%$.

经分析可知, 现有能见度深度学习模型存在 如下问题: (1) 现有方法均采用可见光图像作为输 人, 观测对象数据单一; 其成像原理决定了特征信 息的局限性，无法在不同浓度雾气环境下提供充 分的特征信息. (2) 模型准确率需大数据的支持; 在小样本条件下, 模型训练结果受到网络初始权 值影响, 准确率与鲁棒性均较低.

针对问题(1), 本文分析了可见光-远红外图像 随能见度变化的特征表现. 图 1 给出了不同浓度雾 气环境下, $1 \mathrm{~h}$ 间隔采样的多模态图像灰度直方图 的拟合实例. 其中, 分别在晴朗天气环境下凌晨 4 点和 5 点, 在浓雾天气环境下上午 9 点和 10 点采 样, 其可见光图像及对应的 2 幅远红外图像之间的 灰度直方图拟合实例如图 1a 和图 $1 \mathrm{~b}$ 所示.
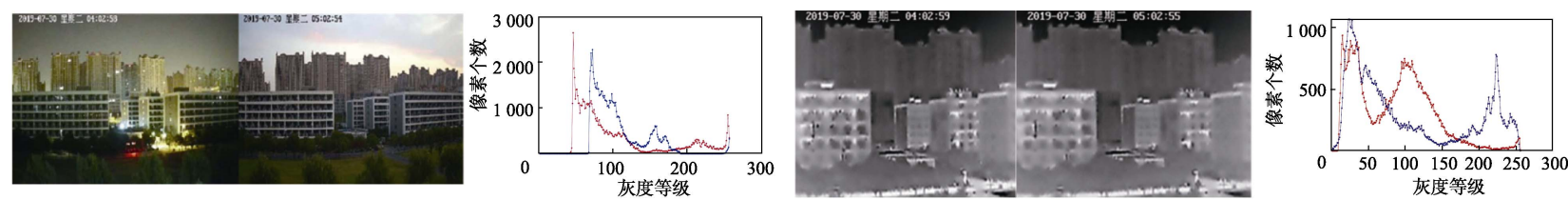

a. 晴朗天气环境下可见光-远红外图像灰度直方图相似度
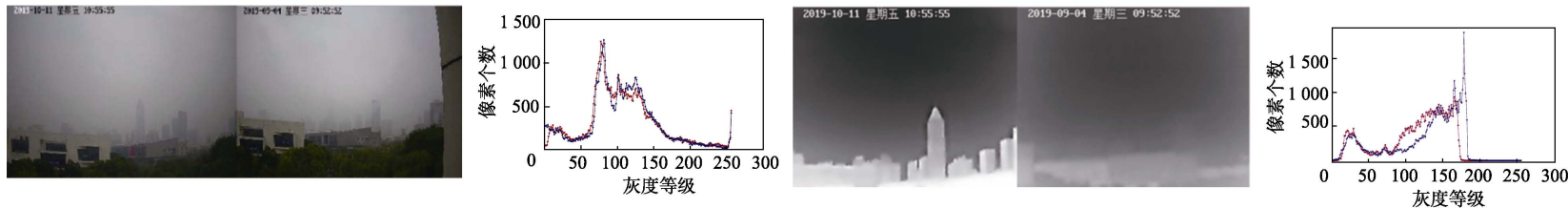

b. 浓雾天气环境下可见光-远红外图像灰度直方图相似度

图 1 不同浓度雾气环境下的可见光-红外图像亮度值响应特性对比分析实例

通过比较不同浓度雾气环境下，可见光、远红 外图像各自的灰度直方图拟合结果可知：远红外 图像在较高浓度雾气环境下, 图像亮度值对于能 见度的变化比较敏感; 相反, 可见光图像在较低浓 度雾气环境下, 其图像亮度值对于能见度的变化 比较敏感. 因此, 可见光-远红外图像对可提供更 加丰富的能见度变化表征特征信息.

针对问题(2), 本文提出了面向可见光-远红外 图像对的多模态能见度检测深度学习模型. 通过 设计多模态特征深度融合网络结构, 有效地融合
各个模态图像的特征信息, 实现模态互补; 在小样 本条件下，提高能见度检测的准确率和鲁棒性.

\section{1 多模态能见度深度学习模型}

\section{1 多模态融合网络框架}

本文提出的深度融合网络模型由可见光图像 特征提取、远红外图像特征提取、叠加特征提取和 特征融合 4 个模块组成. 如图 2 所示, 将可见光和 远红外图像分别输人到 2 个并行的特征提取子网 


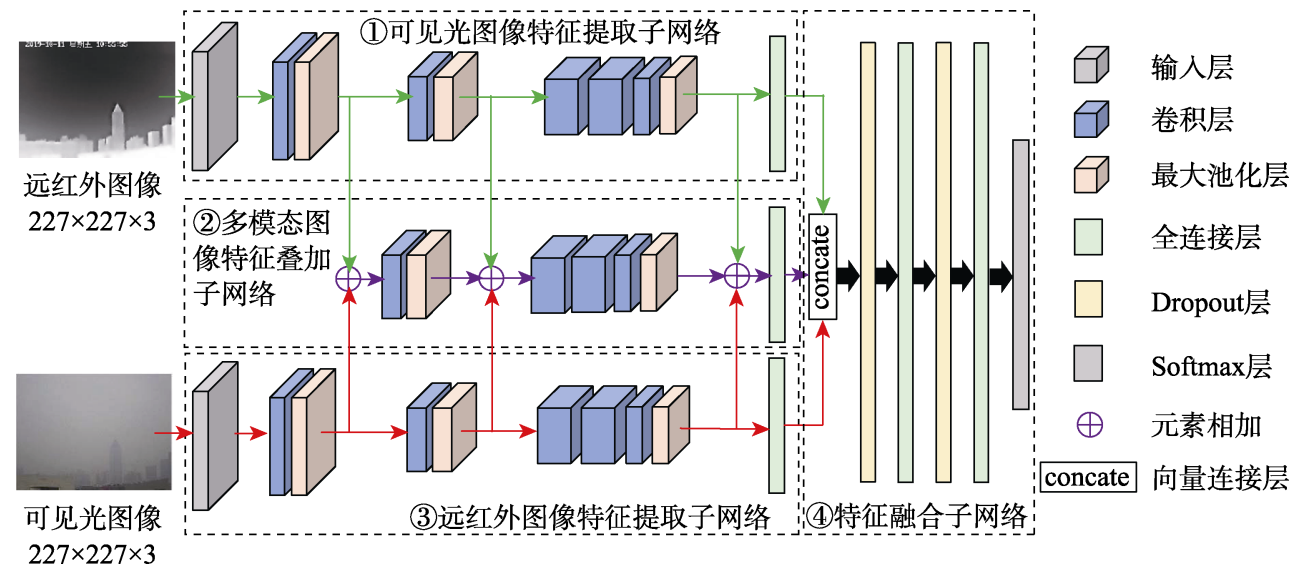

图 2 多模态能见度深度学习网络模型

络, 经过 3 组卷积-池化操作后, 由全连接层映射 到各自的特征空间.

同时, 建立并行特征叠加子网络, 将可见光和 远红外子网络的各池化层输出进行叠加; 再经过 2 组卷积-池化操作后, 由全连接层映射到叠加网络 特征空间. 然后, 将并行各网络全连接层的输出特 征向量进行拼接; 再利用特征融合网络模块, 通过 2 层全连接层增强模型的非线性表达能力, 其过程 中利用 Dropout 抑制过拟合 ${ }^{[16]}$. 最后, 深度融合的 特征向量输人分类器, 完成整个深度融合网络的 特征提取和能见度等级分类过程.

\section{2 可见光、远红外图像特征提取模块}

可见光、远红外图像特征提取模块的网络结构 相同, 包括 5 个卷积层、3 个池化层和 1 个全连接 层. 如图 2 所示, 输人图像大小为 $227 \times 227$ 像素、 3 个通道. 卷积-池化组合的特征提取过程如下: 卷 积层将上层的输出特征图, 利用多个卷积核对其 提取局部特征，获取与卷积核数量相同的特征图. 接着, 将特征图进行最大池化处理、压缩特征, 简 化网络复杂度. 池化后得到的特征图再次作为下 一卷积-池化组合单元的输人, 并逐层向全连接层 方向传递. 这样, 大气特征由局部到整体、由具体 到抽象, 被有效地通过卷积-池化组合操作进行描 述. 其中, 第 $l$ 层的第 $j$ 个特征图矩阵 $\boldsymbol{x}_{j}^{l}$ 由前输出 特征图卷积加权得到, 即

$$
\boldsymbol{x}_{j}^{l}=f\left(\sum_{i \in N_{j}} \boldsymbol{x}_{i}^{l-1 *} \boldsymbol{k}_{i j}^{l}+\boldsymbol{b}_{j}^{l}\right)
$$

其中, $f$ 是神经元激活函数; $N_{j}$ 代表输人特征图 的组合; *表示卷积运算; $\boldsymbol{k}_{i j}^{l}$ 表示卷积核矩阵; $\boldsymbol{b}_{j}^{l}$ 表示偏置矩阵.
本文采用最大池化函数实现图像特征表达的 紧凑性, 增强噪声的鲁棒性 ${ }^{[17]}$.

\section{3 多模态叠加特征提取模块}

为了增强大气特征信息的有效性, 减少对图 像场景理解的偏差. 特征叠加模块将可见光、远红 外图像特征子网络各自第 1 池化层输出的特征图 相加, 获得多模态叠加特征(由于 2 个子网络结构 一致, 特征叠加后其维度不变). 然后, 将叠加特 征输人卷积-池化组合操作进行复合特征提取. 同 理, 在第 2 和第 3 池化层, 将 3 个并行的输出特征 图再次叠加, 并在叠加特征提取模块中向其全连 接层传递. 最后, 获取多模态叠加特征描述向量.

\section{4 多模态特征融合模块}

3 个并行特征提取模块经过多次卷积-池化组 合操作后，原始数据映射到其各自的隐层特征空 间中. 在特征融合模块中, 利用向量拼接将可见光 特征、远红外特征和叠加特征进行连接; 并利用全 连接层将分布式特征映射到样本标记空间, 减少 特征位置对分类带来的影响. 本文所用全连接层 的结构和隐层网络结构相同; 其中, 第 $l$ 层全连接 层的特征向量表示为

$$
\boldsymbol{x}^{l}=f\left(\boldsymbol{w}^{l} \boldsymbol{x}^{l-1}+\boldsymbol{b}^{l}\right)
$$

其中, $\boldsymbol{w}^{l}$ 是权值矩阵; $\boldsymbol{b}^{l}$ 是偏置向量.

为了防止训练过拟合, 在融合模块中添加 2 个 Dropout 层. 模型末端为逻辑回归输出层, 其每个 节点表示输人图片属于某一类别 $i$ 的概率, 表示为

$$
\begin{gathered}
P(Y=i \mid \boldsymbol{x}, \boldsymbol{w}, \boldsymbol{b})=\operatorname{Softmax}(\boldsymbol{w} \boldsymbol{x}+\boldsymbol{b})= \\
\mathrm{e}^{\boldsymbol{w}_{i} \boldsymbol{x}+\boldsymbol{b}_{i}} / \sum_{j} \mathrm{e}^{\boldsymbol{w}_{j} \boldsymbol{x}+\boldsymbol{b}_{i}}
\end{gathered}
$$

其中, $\boldsymbol{w}$ 为权重参数矩阵; $\boldsymbol{b}$ 为偏置矩阵. 


\section{2 实验结果与分析}

\section{1 实验数据采集与标注}

因尚无多模态图像能见度检测公共数据集, 课题组使用双目摄像机在 2019-03-2019-12 于南 通大学收集了不同天气下的室外远景多模态图像 集合, 并利用能见度仪记录同时刻的能见度距离 值对其标注. 图 3 给出了所用设备及输出图像实 例. 其中，可见光原始图像分辨率为 $1092 \times 1080$ 像 素，远红外原始图像分辨率为 $384 \times 384$ 像素.

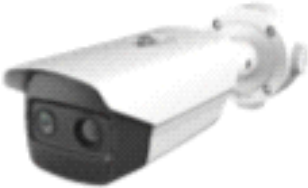

a. 海康威视 DS-2TD2636T-10

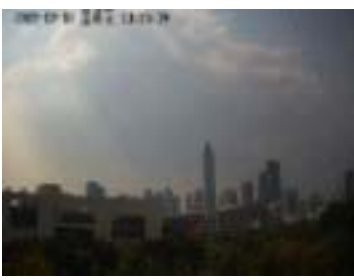

c. 可见光图像

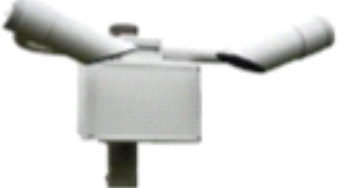

b. 能见度仪 FRT VRE01

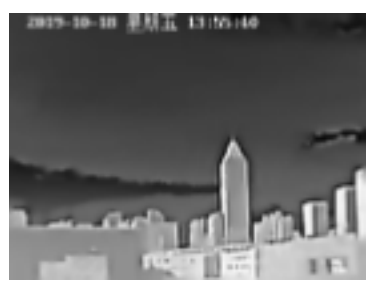

d. 远红外图像

图 3 多模态数据采集标注设备及其输出图像实例

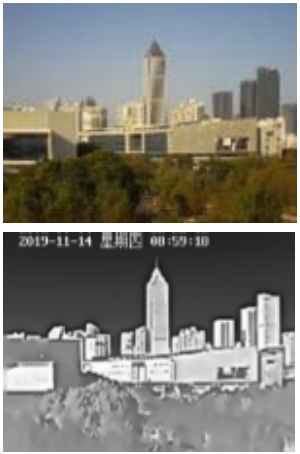

第 1 类
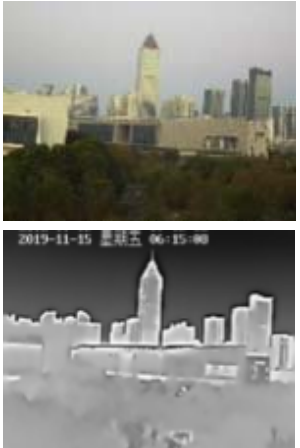

第 2 类

能见度数据收集由天气情况决定，短时间内 较难收集到大范围、等间距的能见度多模态图像. 为了保证每一类内实验图像数量相同, 经过整理, 去除光线过强、曝光不足及高相似度的图像，并参 考能见度距离, 将 2100 幅实验图像集合分成 7 类, 每类各 300 幅图像. 实验数据集随机划分训练集和 测试集各 $50 \%$. 表 1 给出实验数据的分类结果及其 数量. 为方便网络训练, 可见光和远红外图像经配 准，视野和分辨率 $(227 \times 227 \times 3)$ 均相同.

\begin{tabular}{ccc}
\multicolumn{3}{c}{ 表 $\mathbf{1}$ 实验数据 } \\
\hline 分类 & 数量/对 & 能见度值 $/ \mathrm{km}$ \\
\hline 1 & 300 & $5.00 \sim 10.00$ \\
2 & 300 & $2.00 \sim 5.00$ \\
3 & 300 & $1.00 \sim 2.00$ \\
4 & 300 & $0.70 \sim 1.00$ \\
5 & 300 & $0.20 \sim 0.70$ \\
6 & 300 & $0.05 \sim 0.20$ \\
7 & 300 & $<0.05$ \\
\hline
\end{tabular}

图 4 给出了 7 类不同能见度下多模态图像对实 验数据实例. 其中, 每个类别的第 1 行和第 2 行分 别为可见光图像和远红外图像; 且从第 1 类到第 7 类能见度逐渐降低, 雾气浓度逐渐增加.

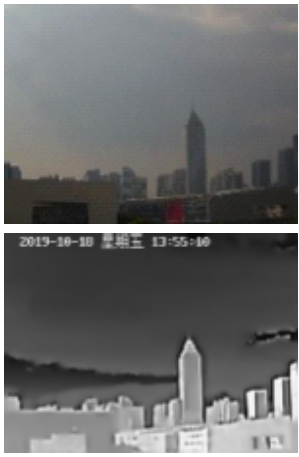

第 3 类

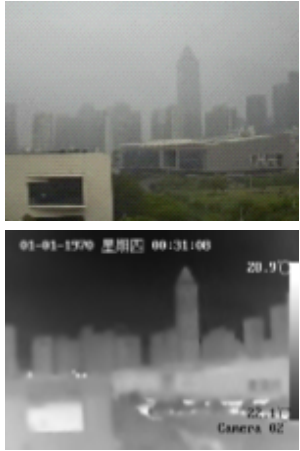

第 4 类

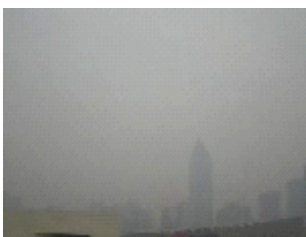

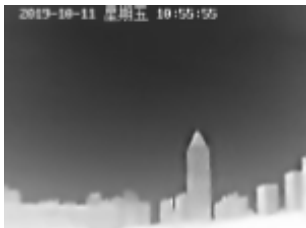

第 5 类

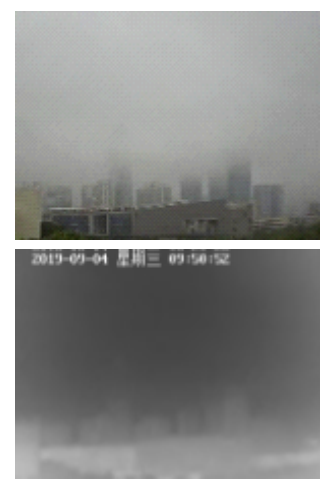

第 6 类

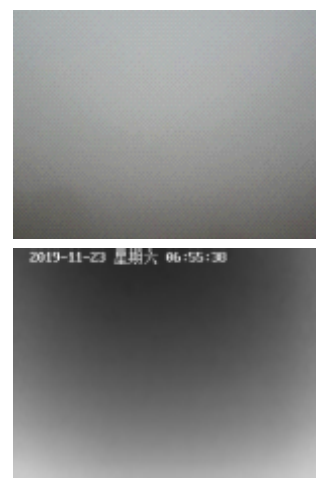

第 7 类

图 47 类多模态能见度图像实验数据实例 


\section{2 实验环境与评价指标}

本文利用 Python3.6 和 Keras 框架完成多模态 能见度模型的构建；服务器环境如下：Windows 10 系统, CPU(Intel Xeon E5-2620), GPU(NVIDIA TITAN xp), RAM(64 GB), CUDA9.2. 通过多组比 较实验验证所提出模型的有效性, 比较对象包括 8
种不同观测数据与网络结构结合的能见度检测模 型: RGB \& AlexNet ${ }^{[18]}$ 模型、IR \& AlexNet ${ }^{[18]}$ 模型、 RGB-IR2 \& AlexNet ${ }^{[18]}$ 模型 (双通道灰度图像), RGB-IR \& AlexNet ${ }^{[18]}$ 模型、RGB-IR \& FusionNet ${ }^{[19]}$ 模型、CNN-RNN ${ }^{[14]}, \mathrm{VisNet}^{[15]}$, 以及本文的多模态 检测模型, 其网络结构参数如表 2 所示.

表 2 多模态能见度检测模型网络参数

\begin{tabular}{|c|c|c|c|c|c|c|c|c|c|}
\hline 结构名称 & \multicolumn{3}{|c|}{ 可见光通道 } & \multicolumn{3}{|c|}{ 远红外通道 } & \multicolumn{3}{|c|}{ 特征叠加通道 } \\
\hline 输人 & \multicolumn{3}{|c|}{$227 \times 227 \times 3$} & \multicolumn{3}{|c|}{$227 \times 227 \times 3$} & \multirow[b]{2}{*}{ 卷积核、数量 } & \multirow[b]{2}{*}{ 步长 } & \multirow[b]{2}{*}{ 输出维度 } \\
\hline 结构说明 & 卷积核、数量 & 步长 & 输出维度 & 卷积核、数量 & 步长 & 输出维度 & & & \\
\hline 卷积层 1 & $11 \times 11 \times 96$ & 4 & $55 \times 55 \times 96$ & $11 \times 11 \times 96$ & 4 & $55 \times 55 \times 96$ & & & \\
\hline BN 层 & & & $55 \times 55 \times 96$ & & & $55 \times 55 \times 96$ & & & \\
\hline 最大池化 & $3 \times 3$ & 2 & $27 \times 27 \times 96$ & $3 \times 3$ & 2 & $27 \times 27 \times 96$ & \multicolumn{3}{|c|}{ Add $27 \times 27 \times 96$} \\
\hline 卷积层 2 & $5 \times 5 \times 256$ & 1 & $27 \times 27 \times 256$ & $5 \times 5 \times 256$ & 1 & $27 \times 27 \times 256$ & $5 \times 5 \times 256$ & 1 & $27 \times 27 \times 256$ \\
\hline BN 层 & & & $27 \times 27 \times 256$ & & & $27 \times 27 \times 256$ & & & $27 \times 27 \times 256$ \\
\hline 最大池化 & $3 \times 3$ & 2 & $13 \times 13 \times 256$ & $3 \times 3$ & 2 & $13 \times 13 \times 256$ & Add $3 \times 3$ & 2 & $13 \times 13 \times 256$ \\
\hline 卷积层 3 & $3 \times 3 \times 384$ & 1 & $13 \times 13 \times 384$ & $3 \times 3 \times 384$ & 1 & $13 \times 13 \times 384$ & $3 \times 3 \times 384$ & 1 & $13 \times 13 \times 384$ \\
\hline 卷积层 4 & $3 \times 3 \times 384$ & 1 & $13 \times 13 \times 384$ & $3 \times 3 \times 384$ & 1 & $13 \times 13 \times 384$ & $3 \times 3 \times 384$ & 1 & $13 \times 13 \times 384$ \\
\hline 卷积层 5 & $3 \times 3 \times 256$ & 1 & $13 \times 13 \times 256$ & $3 \times 3 \times 256$ & 1 & $13 \times 13 \times 256$ & $3 \times 3 \times 256$ & 1 & $13 \times 13 \times 256$ \\
\hline 最大池化 & $3 \times 3$ & 2 & $6 \times 6 \times 256$ & $3 \times 3$ & 2 & $6 \times 6 \times 256$ & Add $3 \times 3$ & 2 & $6 \times 6 \times 256$ \\
\hline 全连接 1 & \multicolumn{3}{|c|}{1024} & \multicolumn{3}{|c|}{1024} & \multicolumn{3}{|c|}{1024 (dropout) } \\
\hline concate & \multicolumn{9}{|c|}{3072} \\
\hline 全连接 2 & \multicolumn{9}{|c|}{4096} \\
\hline Dropout1 & \multicolumn{9}{|c|}{4096} \\
\hline 全连接 3 & \multicolumn{9}{|c|}{4096} \\
\hline Dropout2 & \multicolumn{9}{|c|}{4096} \\
\hline 分类 & \multicolumn{9}{|c|}{7} \\
\hline
\end{tabular}

采用准确率和鲁棒性多角度对各模型的能见 度检测结果进行评价, 其实验过程设计如下：首 先, 对各模型连续训练 5 次, 每次随机设置初始网 络参数; 然后, 统计 5 次训练结果在测试集上的平 均成功率与方差. 在此基础上计算准确率(Accuracy, $A)$ 与鲁棒性(Robust, $R$ ), 其计算公式分别为

$$
\begin{gathered}
A=p_{5} \pm \Delta p_{5} \\
R=\operatorname{var}(\boldsymbol{P}(5))
\end{gathered}
$$

其中, $p_{5}$ 是 5 次训练结果在测试集上的平均成功 率; $\Delta p_{5}$ 是 5 次训练结果在测试集上成功率的浮动 范围; $\boldsymbol{P}(5)$ 为 5 次训练结果在测试集上的成功率 向量; $\operatorname{var}(\cdot)$ 是方差函数.

\section{3 不同模态观测数据的有效性分析}

表 3 给出了 3 种不同的观测数据(RGB, IR, RGB-IR)在相同的 CNN AlexNet 上的能见度检测 实验结果性能比较. 其中，基于可见光 RGB 图像 的能见度检测 5 次平均准确率为 $77.1 \%$; 基于远红
外 IR 图像的能见度检测 5 次平均准确率为 $82.3 \%$; 而基于多模态图像 RGB-IR 的能见度检测平均准 确率最高, 为 $85.0 \%$. 同时, 多模态输人信号的鲁 棒性也最好、方差最小为 0.0000680 . 这说明多模 态图像能够为能见度检测提供更加丰富而有效的 特征信息，并通过融合网络实现“模态互补”。因 此, 其检测准确率和鲁棒性均优于单模态图像.

表 3 不同观测数据的能见度检测模型性能

\begin{tabular}{llc}
\hline \multicolumn{1}{c}{ 模型 } & \multicolumn{1}{c}{$A / \%$} & $R$ \\
\hline RGB \& AlexNet $^{[18]}$ & $77.1 \pm 11.8$ & 0.003457 \\
IR \& AlexNet & $82.3 \pm 4.3$ & 0.000817 \\
RGB-IR \& AlexNet $^{[18]}$ & $85.0 \pm 1.0$ & 0.000068 \\
\hline
\end{tabular}

\section{4 不同融合方式的有效性分析}

在深度学习中, 常见的多模态融合方式分为 信号级、特征级及结果级 3 种融合方式 ${ }^{[20-22]}$. 为验 证本文面向能见度检测提出的多模态特征融合网 
络模型的有效性，将常见的融合方式做了定量比 较与评价.

表 4 所示为基于 2 种多模态融合方式(信号级、 特征级)的 4 种能见度检测模型. 其中, RGB-IR \& AlexNet ${ }^{[18]}$ 和 RGB-IR2 \& AlexNet ${ }^{[18]}$ 均属于信号级 的融合方式，即在 $\mathrm{CNN}$ 提取特征之前，信号端已 经完成多模态融合; RGB-IR2 代表由可见光-远红 外双通道灰度图像输人; 而 RGB-IR \& FusionNet ${ }^{[19]}$ 和本文模型均属于特征级的多模态融合方式. 即, 将 RGB 的彩色图像和 IR 的灰度图像，同时输人 $\mathrm{CNN}$, 通过网络结构实现各模态特征的提取与 融合.

通过比较可知, 对于信号级融合方式而言, RGB-IR 融合方式的性能在准确率和鲁棒性上均优 于 RGB-IR2 双通道灰度图像融合方式. 这说明相 对于灰度图像, 彩色图像的 $R, G, B$ 通道可以为大 气透明度变化的描述提供更有效的颜色信息.

对于特征级融合方式, 2 种网络模型的准确率 均大于 $86.0 \%$, 而信号级融合方式的准确率均小于 $86.0 \%$. 说明通过 CNN 的结构进行特征级别融合 的效果要优于信号级的输人连接融合. 而本文的 融合网络模型在能见度检测的准确率为 $87.2 \%$, 鲁 棒性为 0.00001 ; 充分体现了本文融合网络模型由 浅层至深层对多模态特征进行逐层的“叠加-迭代” 融合的设计, 能够显著地提高输出端能见度联合 特征表征能力的有效性.

表 4 不同网络融合方式的能见度检测模型性能

\begin{tabular}{clcc}
\hline 融合方式 & \multicolumn{1}{c}{ 模型 } & $A / \%$ & $R$ \\
\hline 信号级 & RGB-IR \& AlexNet ${ }^{[18]}$ & $85.0 \pm 1.0$ & 0.00006 \\
融合 & RGB-IR2 \& AlexNet ${ }^{[18]}$ & $84.0 \pm 3.2$ & 0.00032 \\
\hline 特征级 & RGB-IR \& FusionNet ${ }^{[19]}$ & $86.1 \pm 1.1$ & 0.00003 \\
融合 & 本文 & $87.2 \pm 1.0$ & 0.00001 \\
\hline
\end{tabular}

\section{5 图像配准对多模态能见度检测模型的影响}

为增强多模态图像特征提取与融合的效果, 对可见光-远红外图像输人作了配准处理，获取在 视野范围与分辨率均相同的异质图像对. 图 5 所示 为相同的多模态观测数据集在配准前后对应的能 见度检测准确率的变化. 通过比较配准前后的准 确率可知：对于多模态图像输入的能见度检测模 型而言, 无论是信号级的融合、还是利用网络结构 进行的特征级的融合, 输人图像的配准预处理能 够显著地提高其检测准确率.

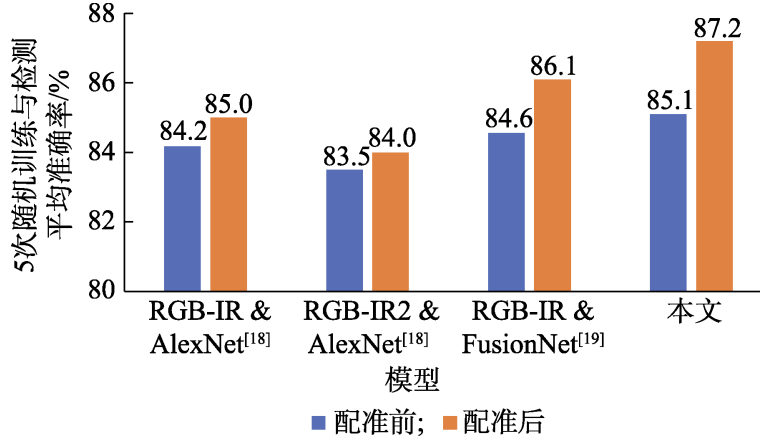

图 5 多模态图像配准对能见度检测模型的影响

\section{6 不同能见度检测模型的有效性分析}

图 6 所示为 8 种能见度检测深度学习模型的 5 次随机初始值训练结果在测试集上的准确率评价 曲线.

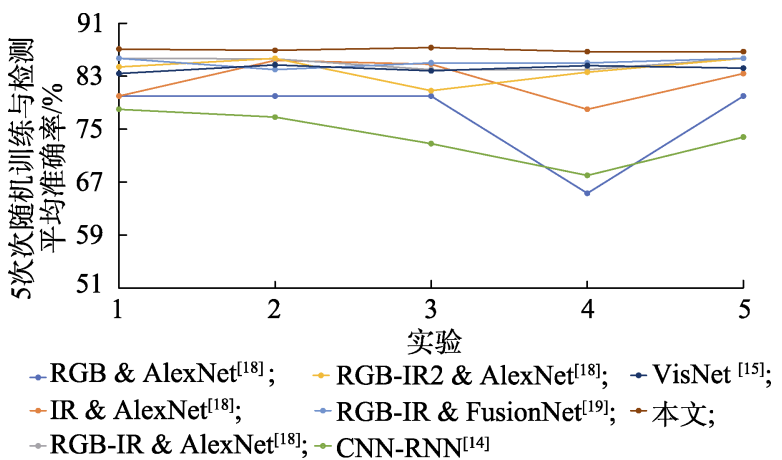

图 6 不同能见度检测模型的性能比较

通过比较可知, 单模态模型的有效性低于其 他多模态模型, 尤其表现在其鲁棒性上. 由于训练 均采用随机网络参数设置, 因此, 其 5 次训练与检 测平均准确率曲线的振荡现象比较突出, 这说明 单模态图像提供的特征稳定性较差, 获取高准确 率需多次训练.

相反，其余多模态模型的振荡现象明显减弱; 经过模态互补, 可见光-远红外图像输人可同时为 能见度检测提供高鲁棒性的特征. 本文的融合网 络无论在准确率和鲁棒性上均取得了最高的性能. 可见, 本文提出的多模态能见度深度学习模型, 比 传统模型可以在小样本的条件下提供更加精确而 稳定的能见度检测准确率.

\section{7 不同模型的训练及检测效率分析}

图 7 所示为 6 种不同模型在训练过程中“准确 率”与 “损失值”的变化曲线. 其中, 黄色垂直虚线 在横轴上的交点代表着模型在验证集上收敛的迭 代次数; 蓝色垂直虚线在横轴上的交点代表着模 型在训练集上收敛的迭代次数. 
比较可知，与其他 3 种融合模型 RGB-IR2 \& AlexNet ${ }^{[18]}$, RGB-IR \& AlexNet ${ }^{[18]}$ 及 RGB-IR \& FusionNet ${ }^{[19]}$ 相比, 本文模型在训练集的准确率与 验证准确率上均迅速收敛、趋于稳定. 而其他 3 种 融合模型的收敛速度相对较慢，这说明本文的融

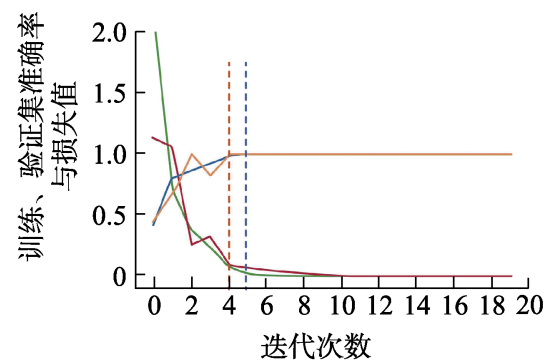

a. RGB \& AlexNet ${ }^{[18]}$

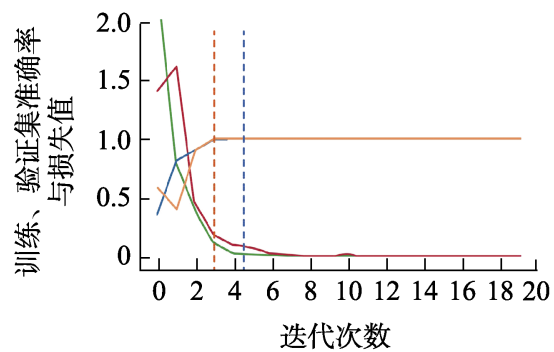

d. RGB-IR \& AlexNet ${ }^{[18]}$

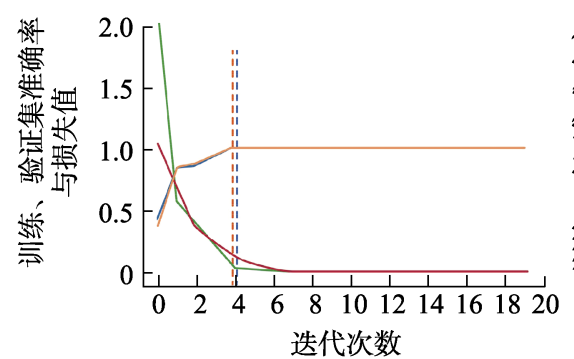

b. IR \& AlexNet ${ }^{[18]}$

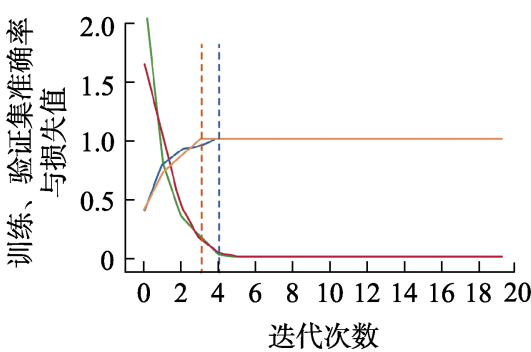

e. RGB-IR \& FusionNet ${ }^{[19]}$

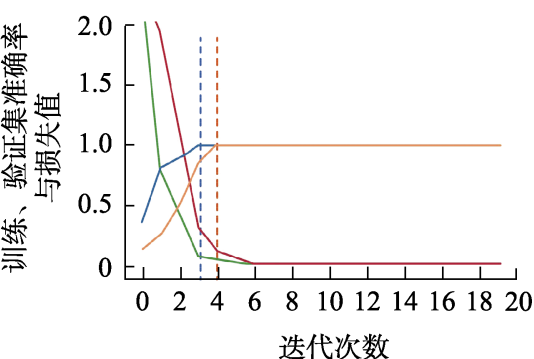

c. RGB-IR2 \& AlexNet ${ }^{[18]}$

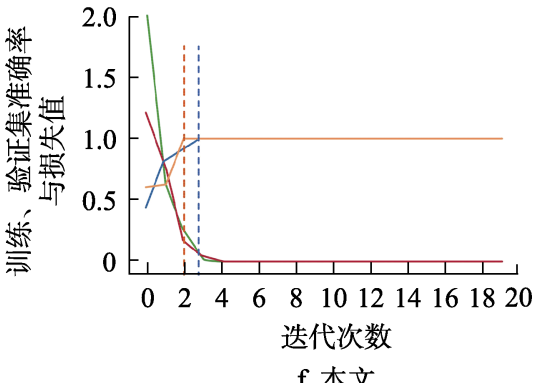

一验证集准确率; 一 训练集准确率; 一 验证集损失值; 一 训练集损失值

图 7 不同能见度检测模型训练过程中准确率与损失值的变化曲线

表 5 所示为不同模型的检测效率，即处理单幅 图像所用的时间. 实验过程设计如下：首先，将训 练好的模型对 100 幅测试图像连续进行能见度检 测; 然后, 求出平均处理每一帧图像的时间. 通过 对比可知, 网络的参数数量由输人信号的通道数 和网络模型的复杂程度决定. 因此, 多模态模型的 网络参数数量明显大于单模态模型, 相对应的模 型的检测图像处理时间也相对略有增加.

\section{表 5 不同能见度检测模型的平均处理时间}

\begin{tabular}{clcc}
\hline 类型 & \multicolumn{1}{c}{ 模型 } & 参数数量 & 时间 $/ \mathrm{ms}$ \\
\hline \multirow{2}{*}{ 单模态 } & RGB \& AlexNet ${ }^{[18]}$ & 58310727 & 22.786 \\
& ${ }^{1 R} \& \mathrm{AlexNet}^{[18]}$ & 58310727 & 22.413 \\
\hline \multirow{5}{*}{ 多模态 } & RGB-IR2 \& AlexNet ${ }^{[18]}$ & 58299111 & 24.963 \\
& RGB-IR \& AlexNet ${ }^{[18]}$ & 58322343 & 24.933 \\
& RGB-IR \& FusionNet ${ }^{[19]}$ & 62058631 & 27.782 \\
& 本文 & 68920199 & 28.478 \\
\hline
\end{tabular}

\section{3 结 语}

本文通过不同观测图像(可见光、远红外)在不 同雾气浓度环境下的亮度值对能见度变化的敏感 程度的分析，得出可见光-远红外多模态图像观测
数据有助于提高图像能见度检测性能(准确率、稳 定性)的推论. 从实现可见光与远红外图像中的大 气特征互补的角度, 提出了面向能见度检测的多 模态特征融合网络模型. 通过设计并行 3 通道浅层 特征提取网络、深层融合网络相结合的方式，弥补 了传统单模态能见度深度学习模型在特征选择和 提取上有效性低的缺点. 实验证明, 多层次、多样 性的特征融合可以显著地提高能见度检测模型的 特征选择能力和分类效果; 同时, 验证了异质图像 配准预处理对于多模态能见度检测模型的性能增 强具有显著效果.

从传感器选择角度来看, 可见光图像可以提 供对能见度检测有益的颜色信息; 而远红外图像 可以提供对能见度检测有益的温度信息. 本文提 出的多模态能见度检测模型, 可以应用于视觉传 感器市场中价格较为低廉的双目摄像机, 对于相 关产品研发起到了相应的引导作用.

\section{参考文献(References):}

[1] Wauben W, Roth M. Exploration of fog detection and visibility estimation from camera Images[OL]. [2020-06-02]. https:// 
www.wmo.int

[2] Negru M, Nedevschi S. Image based fog detection and visibility estimation for driving assistance systems[C] //Proceedings of the 9th IEEE International Conference on Intelligent Computer Communication and Processing. Los Alamitos: IEEE Computer Society Press, 2013: 163-168

[3] Kang J L, Ni R, Andersen G J. Effects of reduced visibility from fog on car-following performance[J]. Transportation Research Record: Journal of the Transportation Research Board, 2008, 2069(2): 9-15

[4] Xu Min, Zhang Hongying, Wu Yadong. Image visibility detection algorithm based on scene depth for fogging environment[J]. Process Automation Instrumentation, 2017, 38(9): 89-94(in Chinese)

(徐敏, 张红英, 吴亚东. 基于场景深度的雾天图像能见度 检测算法 [J]. 自动化仪表, 2017, 38(9): 89-94)

[5] Varjo S, Hannuksela J. Image based visibility estimation during day and night[C] //Proceedings of Asian Conference on Computer Vision. Heidelberg: Springer, 2014: 277-289

[6] Hautiére N, Babari R, Dumont É, et al. Estimating meteorological visibility using cameras: a probabilistic model-driven approach[C] //Proceedings of the Asian Conference on Computer Vision. Heidelberg: Springer, 2010: 243-254

[7] Jeevan S, Usha L. Estimation of visibility distance in images under foggy weather condition[J]. International Journal of Advanced Computing and Electronics Technology, 2016, 3: 11-16

[8] Zhu L, Zhu G D, Han L, et al. The application of deep learning in airport visibility forecast $[\mathrm{J}]$. Atmospheric and Climate Sciences, 2017, 7(3): 314-322

[9] Chaabani H, Kamoun F, Bargaoui H, et al. A neural network approach to visibility range estimation under foggy weather conditions[J]. Procedia Computer Science, 2017, 113: 466-471

[10] Hautiére N, Tarel J P, Lavenant J, et al. Automatic fog detection and estimation of visibility distance through use of an onboard camera[J]. Machine Vision and Applications, 2006, 17(1): 8-20

[11] Babari R, Hautière N, Dumont É, et al. A model-driven approach to estimate atmospheric visibility with ordinary cameras[J]. Atmospheric Environment, 2011, 45(30): 5316-5324

[12] Li Q, Li Y, Xie B. Single image-based scene visibility estimation[J]. IEEE Access, 2019, 7: 24430-24439

[13] Li S Y, Fu H, Lo W L. Meteorological visibility evaluation on webcam weather image using deep learning features[J]. Inter- national Journal of Computer Theory and Engineering, 2017, 9(6): 455-461

[14] Yang Y, Lu C W, Wang W M, et al. Relative CNN-RNN: learning relative atmospheric visibility from images[J]. IEEE Transactions on Image Processing, 2019, 28(1): 45-55

[15] Palvanov A, Cho Y I. VisNet: deep convolutional neural networks for forecasting atmospheric visibility[J]. Sensors, 2019, 19(6): 1343

[16] Wei Fan, Song Yunfei, Shao Mingli, et al. Improving adversarial robustness on single model via feature fusion and ensemble diversity[J]. Journal of Software, 2020, 31(9): 1-14(in Chinese) (韦璠, 宋云飞, 邵明莉, 等. 利用特征融合和整体多样性提 升单模型鲁棒性 [J]. 软件学报, 2020, 31(9): 1-14)

[17] Zhang Shun, Gong Yihong, Wang Jinjun. The development of deep convolution neural network and its applications on computer vision[J]. Chinese Journal of Computers, 2019, 42(3): 453-482(in Chinese)

(张顺, 龚怡宏, 王进军. 深度卷积神经网络的发展及其在 计算机视觉领域的应用 [J]. 计算机学报, 2019, 42(3): 453482)

[18] Krizhevsky A, Sutskever I, Hinton G E. ImageNet classification with deep convolutional neural networks $[\mathrm{J}]$. Communications of the ACM, 2017, 60(6): 84-90

[19] Eitel A, Springenberg J T, Spinello L, et al. Multimodal deep learning for robust RGB-D object recognition[C] //Proceedings of the IEEE/RSJ International Conference on Intelligent Robots and Systems. Los Alamitos: IEEE Computer Society Press, 2015: 681-687

[20] Sanchez-Riera J, Hua K L, Hsiao Y S, et al. A comparative study of data fusion for RGB-D based visual recognition[J]. Pattern Recognition Letters, 2016, 73: 1-6

[21] Lin Suzhen, Han Ze. Images fusion based on deep stack convolutional neural network $[\mathrm{J}]$. Chinese Journal of Computers, 2017, 40(11): 2506-2518(in Chinese)

(济素珍, 韩泽. 基于深度堆叠卷积神经网络的图像融合 [J]. 计算机学报, 2017, 40(11): 2506-2518)

[22] Lu Hongyu, Zhang Min, Liu Yiqun, et al. Convolution neural network feature importance analysis and feature selection enhanced model[J]. Journal of Software, 2017, 28(11): 2879-2890 (in Chinese) (卢泓宇, 张敏, 刘奕群, 等. 卷积神经网络特征重要性分析 及增强特征选择模型[J]. 软件学报, 2017, 28(11): 2879-2890) 